\title{
Designing Indoor Tangible Games based on Fuzzy Localisation
}

\author{
Raphaël Robert-Bouchard, Jérôme Dupire, Pierre Cubaud \\ CEDRIC - Conservatoire National des Arts et Métiers \\ 292 rue St Martin, 75003 Paris, France \\ raphael_rb@hotmail.fr,\{dupire, cubaud\}@cnam.fr
}

\begin{abstract}
We present in this paper the development of an easy-to-adapt architecture for radio based, indoor geolocation; and the new opportunities it brings for game design. The weak precision of such systems, usually regarded as a problem, is considered here as a source of innovation for the design of pervasive games.
\end{abstract} design

Keywords - geolocation, zigbee, beacon, location-based, game

\section{INTRODUCTION}

The IEEE 802.15.4 standard allows for wireless connections of sensor networks at a range of several dozen meters. These networks were also used to support indoor geolocation attempts. But like other conventional radio protocols (WiFi, Bluetooth), it does not currently achieve high accuracy (i.e., close to the GPS outdoor's one). Usually considered as a weakness, this inaccuracy has been integrated into this project as the very component that could be used to bring innovation. Our aim was to design an indoor locationbased game, whose core gameplay would exploit this uncertainty inherent to the system. The proposed design space is between the board game and outdoor location based game. The advantages are the appropriation or re-appropriation of a known environment by turning it into a playground. Ultimately, we'll reach ease of deployment and the almost immediate launch of the game, since our environment will naturally be populated with communicating devices (smart objects, connected TVs, etc.) that may provide usable infrastructure to our system. After a review of previous works, we present the hardware architecture that we designed. Then we will describe the Scuba World game, developed with a specific configuration of the previous architecture. We then present the results of an evaluation experiment conducted for this game, before concluding.

\section{RELATED WORK AND BACKGROUND}

\section{A. Location-based Game Design}

Many outdoor location-based games have been studied in the scientific literature since the early 2000s. Can You See Me Know? [1] is a hunting game. Up to 15 players are pursued by three professional riders throughout the city. Hunted people "exist" only in a virtual world, whereas the runners, located by GPS, must physically go to the right location to catch them. Mogi [2] is a treasure search game. Placed by the game designers, treasures appear regularly in a virtual space, and the only way for the player to recover the treasure is to physically move through the city, located by a GPS system. Once in place, the player can collect the object. On his way, he will meet other treasure hunters with whom he can communicate, socialize and exchange treasures. Savannah [3] is the most cited educational location-based game on Google Scholar. Using a PDA, the game turns a school playground into a virtual African Savannah. Using sound and images from the real Savannah, the game brings players to act like a real lion pack to mark their territory and hunt prey. Ingress [4] is an augmented reality game created by Google. In this massively multiplayer game, the players are split into two camps and must conquer territories. These territories are pieces of the real environment and players must travel to strategic keypoints in the real world in order to fight for their control. Here geolocation is also done via GPS. Besides the fact that they use geolocation in their gameplay, these games share a common feature: they are all outside. The vast majority go through the GPS to allow accurate geolocation. However, there are lessons to be gained from these games, despite the difference in scale, as shown by a section on lessons learned from the analysis of geocaching done by Neustaedter et al. [5]. Geocaching is an activity becoming increasingly popular over the years. Halfway between sport and gambling, geocaching is a treasure hunt in the world, consisting of 2 phases, theoretically independent. The cache phase consists of creating a "geocache" a box which can vary in form and content. Often (not always), it contains a notebook and pencil. This box is then hidden at a precise location, and whoever conceals it notes the precise GPS coordinates of the location. The searcher then goes on the geocaches website and publicly reveals the coordinates. The other phase of the game is the search. A person will try to find a geocache in coordinated it has recovered from the referral sites. The Geocache is usually well hidden. To go to the coordinates is often insufficient to find the object. Once the person has found it, she will write her name and the date in the associated diary and will put the object back in place to allow other players to do their own research. Neustaedter et al. [5] chose to study this game because, after 14 years of existence, it is the only location-based game that managed to be sustainable and continue to see its number of users growing. Now, apart the Ingress beta, no other location-aware game has survived for more than 2 years. Having studied and even participated in the operation of geocaching for a year, they reached several interesting conclusions. First the authors have learned lessons in relation to games of the same type and same scale. Then, they extracted the general characteristics to differentiate types of location-based games: the type of usable game location, the 
complexity for the content of the game, the timing of the interaction between players and finally the type of entity that created the content of the game. These characteristics are quite close to those set out in [3] about location-based games. Although rare, some studies exist about the technical limitations of indoor location-based games, especially in terms of time and accuracy. In 2012, the most frenetic action game using geolocation, Sword fight, was created to repel these technical limitations [6]. There was a delay of a hundred millisecond minimum, giving the illusion that the system response was instantaneous. This system was accurate to the centimeter, even at a speed of 2 meters per second.

\section{B. Uncertainty and Game Design}

The object of research here is the spatial uncertainty as a gameplay catalyst. This uncertainty is the fact that the player has some doubts on certain borders in the game space that she's practicing. As far as we know, very little research has been published on this subject. Two papers have yet addressed the spatial uncertainty in the game, each approaching the concept in a radically different angle. In both cases, the games are some kind of game of cat and mouse using the GPS system and using the same standard: IEEE 802.11. In [1] Benford et al. consider the spatial uncertainty as a defect that must be managed. Uncertainty can be reduced by technology or by the game design, because some of its effects can be hidden to the player. On the contrary, her attention can be drawn on it, for her to take note of this uncertainty as something external to the game that she will have to deal with. In all cases, the aim is to manage the problem early, during the creation of the game and setting it up so that it interferes as little as possible in the users gaming experience. Chalmers et al. [7] take a very different approach. GPS can sometimes have gray areas or "cold spots". In these places the player cannot be positioned using GPS. Addressing this phenomenon positively, the authors have experienced a game with two teams of players are made. Each team must pick up in the same neighborhood some coins scattered on a virtual map corresponding to the physical topology. Each player tries to steal the other players or to protect herself. Using a PDA, each player knows the position of the coins, of the other players, and the signal strength levels in the area. A possible strategy is then to lie in ambush, voluntarily in an area where GPS does not work, attempting to surprise a player and steal it. Although very short, this article has the merit of showing that a game can be developed based on the idea of using a technological "defect". We think that it can also be interesting for indoor games.

\section{ARCHITECTURE}

We describe here our generic architecture that allows for a fuzzy geolocation feature. The benefits of our proposal are a low cost and ease of implementation. This architecture consists of two objects: the beacons, on the one hand and the one or more mobile devices, on the other.

\section{A. Geolocation methods}

Range-based techniques typically exploit the Received Signal Strength Indication (RSSI) and the flight time while the range-free solutions estimate the location of sensor nodes by, either, exploiting the radio connectivity information among neighboring nodes, or exploiting the sensing capabilities that each sensor node possesses. Due to the distinct characteristics of these two approaches, we categorize the range-free localization schemes into: anchor-based schemes (which assume the presence of sensor nodes in the network that have knowledge about their location) and anchor-free schemes, which require no special sensor nodes for localization. Our proposal belongs to the category of range-free solution (anchor-based). In this context, several technologies are naturally candidates: WiFi, Bluetooth (BT), ZigBee (ZB). WiFi present best performances in terms of throughput and range. This is however not the criteria that interest us. Indeed, a low cost and a reduced range were privileged. The WiFi was thus not retained. The short range of other solutions mentioned above (BT, ZB) allows us to consider reduced radiation areas for each anchor, and be consistent with the scale of the areas identified through our system. To choose between these two technologies, we considered the energy consumption criterion, since all of the objects should be battery powered. We therefore choose the ZB solution.

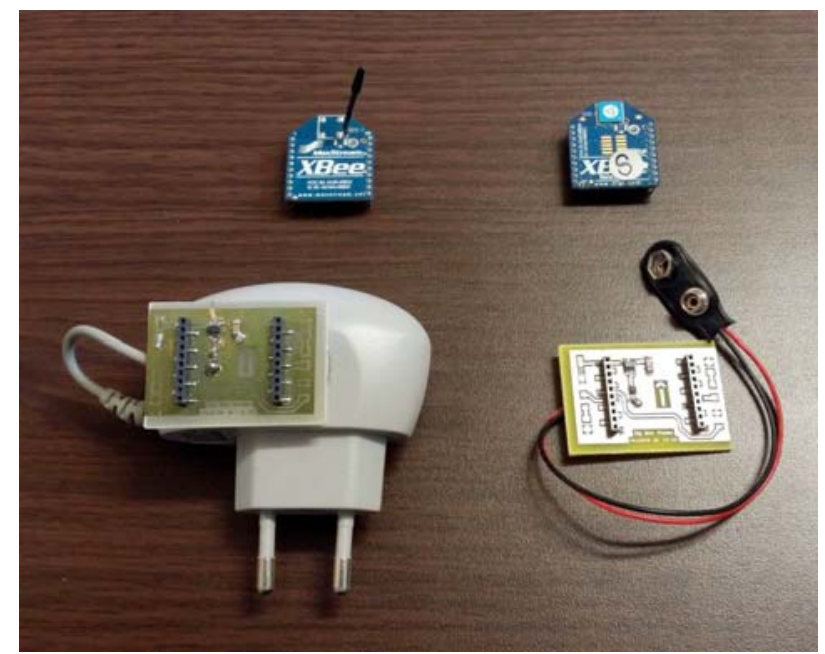

Fig. 1. ZigBee modules (XBee series 1 from Digi) wire antenna (top left) and chip antenna (top right) and PCB with power supply: wall plug revamped from a mobile phone (bottom left) and battery powered (bottom right)

Our approach differs insofar as our anchor network is not spatially fixed by the constraints of associated sensor architecture. It will not support any useful communication. This allows us to position each anchor freely. Their positioning will only be driven by the needs identified in the game design (i.e., the deployment of gaming areas in the real environment).

\section{B. Beacon}

In our architecture, the anchor is equipped with a $\mathrm{ZB}$ module (XBee Series 1 from Digi) and is powered either by a battery or by a wall plug (Fig.1). This alternative allows us to be completely independent of the presence of electrical outlets in the choice of the anchors location. The electronics consists of a simple voltage regulator with smoothing capacitors. We use only one functionality offered by the radio communication protocol: a particular node can send (broadcast) a request in order to make all the nodes within the emitting range respond 
(AT ND command: Node Discover). All nodes receiving this packet will always respond with a message containing some information about them, such as their MAC addresses or label. By periodically sending the AT ND command, one is thus able to know what nodes are within reach of the mobile node and to deduce relative positioning, as described in Fig. 2.

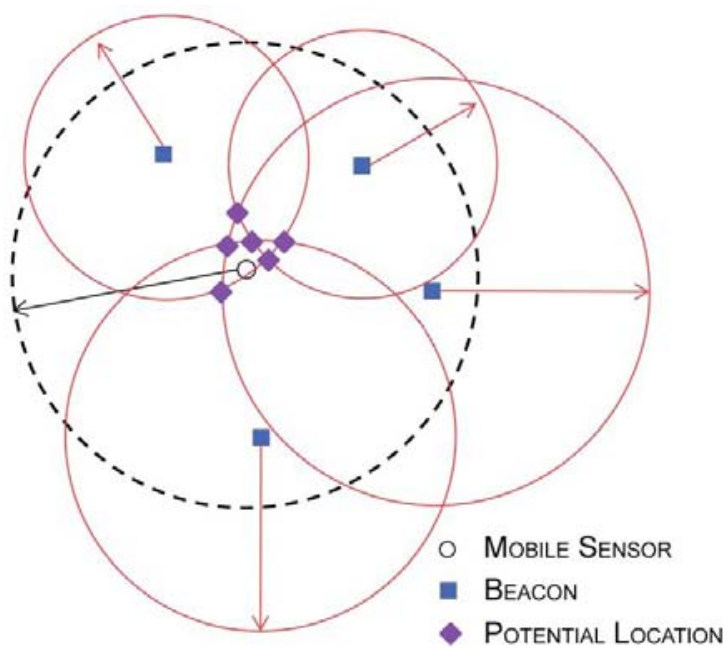

Fig. 2. Range-free localisation (with beacons)

Finally, two different types of modules, associated with two types of antennas have been used: the modules with a wire antenna (Fig.2 top left) and the modules with a chip antenna (Fig.2 top right). This difference allows us to adapt the different scopes of the two types of antenna to the context of the deployment, including the surface of the global game area and the game sub-areas to be delimited.

\section{Mobile Device}

The mobile device is the object that will be the interface between the game and the player. It is based on an Arduino UNO board, a ZB shield and a Xbee series 1 chip (from Digi). This device hosts a program on the one hand, triggering periodic emissions of the AT ND command and the treatment of the responses received (i.e., the comparison of the beacons that responded with a topographic model of the organization of these beacons) and, on the other hand, manages the user interface (UI). This UI can consist of LED's, or 7 segments LCD display, or any other device or combination of device manageable by the embedded microcontroller.

\section{THE SCUBA World GAME DESIGN}

\section{A. The Game}

The game can be played in a single room or inside an apartment / house. It is a two-player game. One player hides a few objects, "pearls" to find for the second player, and then places the beacons to define some "air bubbles". Pearls are small physical objects, unrelated to the electronic system and there are 6 of them to find during a game session. The air bubbles are invisible areas allowing the second player (called the fisherman) to breathe.

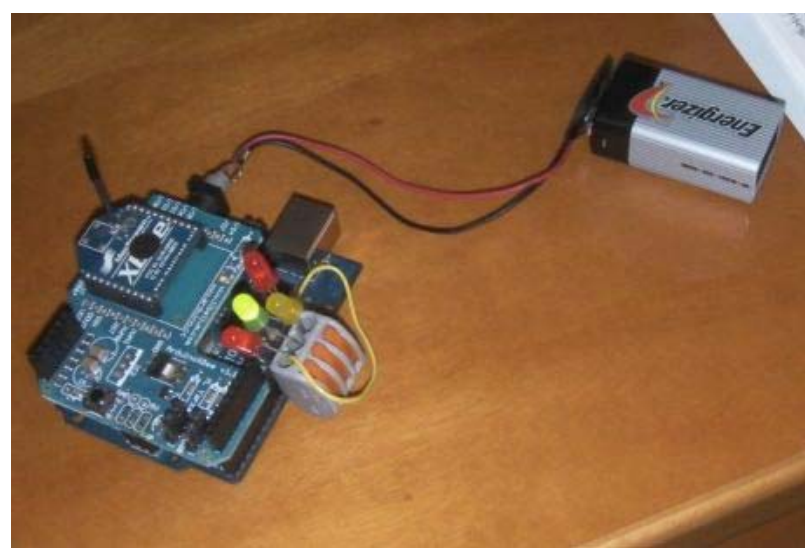

Fig. 3. The mobile device used in the Scuba World game

On a second time, the purpose of the fisherman is to find the pearls scattered in the play area, while taking care not to choke. He has indeed a limited air supply when searching the room. To restore his air supply, he must return regularly in bubbles located in the room. The bubbles are areas of superposition of the radiation range of the 3 beacons. When the fisherman is in a bubble, he can stay as long he wants, and his air gauge fills up in 3 seconds. When the fisherman is out of this air bubble, he is considered snorkeling and air supply decreases. A full air supply makes the player autonomous for 1 minute and 35 seconds. After this duration, without any resupply, the player dies (game over). The metaphor of fishing and snorkeling was chosen for several reasons. First of all, it is a simple metaphor that speaks to everybody in terms of opportunities and constraints. It has also been long used in video games. In addition it helps the player to conceptualize this 3D space, the more or less shaped bubbles, with which he/she will interact regularly. And finally because the radio waves tend to be fluctuating [8], as could be an air bubble lightly tossed by seabed.

\section{B. Hardware}

The object that the fisherman player holds during the game is a plastic box of $8 \mathrm{~cm}$ wide, $15 \mathrm{~cm}$ long, $6 \mathrm{~cm}$ high (Fig.3) containing all the electronics. The upper side is open, allowing the user to see the color diodes (LED). There are 4 LED: a yellow, two red and one green. The yellow LED serves as an alarm: when there are only 15 seconds left for the player to breath, it turns on. When the player has totally exhausted her air supply, it flashes, signifying the end of the game. The three other diodes are used for the identification of air bubbles. Each beacon is associated with a diode. When the mobile device is within the range of a beacon, the corresponding LED turns on. When the three LEDs are switched on, the player is in a bubble and its reserve fills up. There is no display for the remaining air. The player must estimate by herself the time she can remains away from the air bubble. The solid yellow LED is the only (and late) warning for her to know. 


\section{EXPERIMENT}

The game Scuba World was tested in order to verify if there was some fun playing with it, and more generaly with rules based on spatial uncertainty.

\section{A. Subjects, settings and protocol}

Twenty unpaid adults participated to the experiment (mean age: 36.7 years, standard deviation $\sigma=11.8$ ), including 11 men and 9 women. All were students or staff from our university. Most of them don't work in a field related to game and none of them played a location-based game before the experiment. The tests were conducted in a (messy) laboratory office that measures $3 \mathrm{~m}$ wide and $5 \mathrm{~m}$ long. The briefing prior to the game session took place in a nearby office. The three beacons and the pearls were always placed in the same locations, in order to present the same initial situation to all participants. The beacons' position lead to the creation of an overlapping area in the center of the room. This configuration should cause the player to explore the periphery of the room, forcing him to get out of this bubble regularly. The pearls were hidden differently (from easy to difficult) allowing the player to discover them during the 10 minutes experience. Each participant played the game alone. The experimenter simulated the second player, by placing in advance the tags and pearls. The experimenter explained the game context to the participants and the rules: duration of apnea, air bubble system and the LED meanings. It was specified, since the prototype is radio-based, that the body can act as a screen and therefore sometimes a slight displacement of the player's body could have consequences on radio communication between beacons and the mobile device. The experimenter placed the player with the mobile device in the main air bubble. Then the game began. After 10 minutes, or if the player "died" before this time, the game ends. The player was then asked to answer a short questionnaire and then performed a self-confrontation, during the presentation of the session's recorded video.

\section{B. Results}

The purpose of the questionnaire was to have a feedback regarding the gaming experience. Desire to replay the game was graded on average $7.0(\sigma=1.7)$ and game fun was graded $6.8(\sigma=1.5)$, on a scale ranging from 1 (weakest) to 10 (strongest). Only 3 participants gave a rating of 4 to one of these two questions, and no lower rating was given. Fourteen participants wanted to place the pearls for the next player. This would tend to indicate that the game was generally appreciated by the participants. Eight participants reported they would recommend the game to everyone, and 8 specifically to children. Eight participants slightly underestimated and 5 participants slightly overestimated their play time. During the play sessions, participants were silent most of the time. However, during self-confrontation, most participants spoke spontaneously. The first topic discussed was often the air bubble strategy. For the 20 participants, many tactics have emerged. Some players have found those bubbles too small, others were more confident in their exploration once they knew for sure how to find a bubble. Four players preferred to neglect other potential bubbles to focus on one. One of them argued that due to the relatively small size of the room, he did not have to bother with another bubble than the one he knew. Conversely, 3 players have specifically sought several points of folds, often taking one that seemed closest to them. The others were more opportunistic, seeking a bubble if they thought they could find one near, if not back to the initial location. Overall, it seemed easy for the player to conceptualize this virtual space of air bubbles, or at least the rules to play with. The link between this virtual space and the player was the mobile device. This object has a very special meaning for the participants and it appeared difficult to most of them to drop it when looking for the pearls. Only 2 participants left the device during their session. Participants also noted the system stability problems (i.e., the fuzzy part). Because of the anisotropic behavior of waves, the areas covered by the beacons could fluctuate, which prompted most participants to be careful. The limits of the air supply have been reached only by one player.

\section{CONCLUSION AND FUTURE WORK}

Uncertainty is at the core of any game. This project shows that a new form of uncertainty - spatial uncertainty - can be exploited when designing games. The indoor location system presented here can be developed at a low cost and in a very short time, opening up new opportunities for game designers. The design, development and testing of the game Scuba World demonstrate the technical feasibility and the innovative nature of this type of experience for the players. Today, radio networks constantly run around us, in our homes or in public buildings. By considering networks irregularities as a design option and combining it to connected objects, one can open a whole new area for game creation and play. For enhenced game experience, we now need to imagine objects, like pawns or even bigger toys, and design them in an adaptable way.

\section{REFERENCES}

[1] Benford S., Anastasi R., Flintham M., Drozd A., Crabtree A., Greenhalgh C. 2003. Coping with uncertainty in a location-based game. IEEE Pervasive Computing, vol. 2, (3), pp. 34-41.

[2] Licope C., Inada Y. 2005. Les usages émergents d'un jeu multijoueurs sur terminaux mobiles géolocalisés : les conséquences interactionnelles des “rencontres d'écran". UBIMOB 05, May 31.

[3] Reid J. 2008. Design for coincidence : Incorporating real world artifacts in location based games. DIMEA'08, September 10-12.

[4] Hodson H. 2012. Google's Ingress game is a gold mine for augmented reality. New Scientist, vol. 216, no 2893, p. 19.

[5] Neustaedter C., Tang A., Judge T. K. 2011. Creating scalable locationbased games: lessons from Geocaching. Springer-Verlag London Limited 2011.

[6] Zhang Z., Chu D., Chen X., Moscibroda T. 2012. SwordFight: Enabling a New Class of Phone-to-Phone Action Games on Commodity Phones. MobiSys'12, June 25-29, 2012.

[7] Chalmers M., Bell M., Hall M., Sherwood S., Tennent P. 2004. Seamful Games. Computing Science, University of Glasgow.

[8] Ganesan D., Krishnamachari B., Woo A., Culler D., Estrin D., \& Wicker S. 2002. Complex behavior at scale: An experimental study of lowpower wireless sensor networks. Technical Report UCLA/CSD-TR 02. Vol. 13, pp. 1-11. 